\title{
Spontaneous Escherichia coli persisters with week-long survival dynamics and lasting memory of a short starvation pulse
}

\author{
Mikkel Skjoldan Svenningsen ${ }^{1}$, Sine Lo Svenningsen², Michael Askvad Sørensen ${ }^{2, *}$, Namiko Mitarai ${ }^{1, * *}$
}

\begin{abstract}
The vast majority of a bacterial population is killed within a time scale comparable to their generation time when treated with a lethal concentration of antibiotics. However, a small subpopulation typically survives for an extended period. To investigate the long-term killing dynamics of bacterial cells we constructed a week-long killing assay and followed the survival fraction of Escherichia coli K12 exposed to a high concentration of ciprofloxacin. We found that long-term survivors were formed during exponential growth, with some cells surviving at least 7 days. In contrast to the biphasic killing dynamics typically used to describe persistence, the long-term dynamics contained at least three timescales, which greatly enhances predictions of the population survival time. Furthermore, we observed a surprisingly long memory effect of a brief starvation pulse, which was dependent on the (p)ppGpp synthase relA. Specifically, one hour of carbon starvation prior to antibiotics exposure increased the surviving fraction by nearly 100 -fold even after 4 days of ciprofloxacin treatment.
\end{abstract}

Importance: Bacterial infections pose an increasing worldwide health risk due to the rise of antibiotics resistance. This motivates the question of how to administer antibiotics while minimizing the time the bacteria are exposed to the antibiotic to reduce the development of resistance, and poses a need for understanding bacterial killing dynamics. We investigate the long-term time-dependent survival of $E$. coli during one week of exposure to ciprofloxacin. We demonstrate that a subpopulation of bacteria survived for several days even when ciprofloxacin was added to exponentially growing cultures. Furthermore, a short starvation pulse resulted in a long-lasting effect on survival. The revealed killing dynamics show that long-term persistence is not caused by simply one subpopulation of antibiotic-tolerant cells, but is a complex, time-dependent phenomenon.

Keywords: Bacterial persistence, Stress response, Growth physiology, Antimicrobials, (p)ppGpp

\section{Introduction}

Bacterial populations are quickly decimated during a typical antibiotics assault. Within a few generation times, the far majority of cells will be dead. However, it is typically recommended to use extended durations of treatment, ranging from several days to months, prolonging the exposure time of bacterial pathogens to the antibiotic [1]. The WHO is now considering the benefits of shortening the duration of antibiotics administration while still keeping the treatment effective, due to concerns of increasing antibiotic resistance occurring as a

\footnotetext{
*correspondence: mas@bio.ku.dk

** correspondence: mitarai@nbi.ku.dk

${ }^{1}$ The Niels Bohr Institute, University of Copenhagen

${ }^{2}$ The Department of Biology, University of Copenhagen
}

12 consequence of increased exposure [1]. To find the op3 timal treatment duration, one needs to understand the killing dynamics of bacteria when exposed to antibiotics, especially the bacterial cells surviving for longer times. The long-term survivors are typically referred to as persister cells, a subgroup of cells that survive antibiotics for an extended period compared to the average of the population, but have not acquired mutations that make them resistant to the antibiotic [2, 3]. Most research on persister cells is done within the well-defined conditions of the laboratory, but despite these strongly simplified conditions, and more than seventy years of research, laboratory persisters are still far from understood $[4,5]$.

One pending question is whether and how much persisters form spontaneously during the exponential growth phase. Such persisters are called type-II [3] or 
spontaneous [4] persisters. It was repeatedly shown that 81 stress-triggered (or type I) persisters are formed in high 82 numbers during the stationary phase, but research on spontaneous persister formation during the exponential phase is sparse [4]. The research has mostly been confounded by a lack of careful attention to the presence of stationary phase cells carried over from the starter cultures, which artificially elevated the persister fraction of exponential cultures $[5,6,4]$. One carefully executed study showed that no $E$. coli persister cells were formed during fast exponential growth in rich medium [7], whereas other studies merely showed reduced levels during exponential growth $[3,5]$. A benefit of analysing the exponential growth phase is the well-defined physiology of this state $[8,9]$. This makes it possible to vary the growth physiology in a controlled manner, especially by varying the growth rate through culturing bacteria in media of different nutrient quality. It was previously shown that the bacterial growth rate strongly correlates with the death rate during the initial period of killing with beta-lactams $[10,11,12]$. This poses the additional questions of how the growth rate at the time of antibiotics exposure affects the short- and long-term killing dynamics.

The current standard for persister identification at the population level is a biphasic killing curve, where two time-scales are identified in the time-kill curves [4]. ${ }_{107}$ Persisters are identified as the subpopulation with a sec- ${ }_{108}$ ond, slower killing rate than the rapid death rate of the ${ }_{109}$ primary population. If only two timescales are present ${ }_{110}$ in the killing dynamics, the population survival time can ${ }_{111}$ be extrapolated from the second slow killing rate. Notably, a few studies have reported deviations from the biphasic model. In one case, a heavier tail of longterm survivors was observed, possibly due to low level ${ }_{11}$ contamination of the persister assay [13]. In another case, a powerlaw tail in the short-term killing dynamics was observed [14]. Altogether, the long-term survival of the antibiotics-tolerant subpopulation is gravely understudied. Most in vitro lab research on persisters of fast growing bacteria as $E$. coli is carried out for three to five hours $[15,7,5]$, though some studies increase the exposure time to $24-50$ hours $[2,16,17,3]$. Investiga- 120 tion of long-term survival beyond the typical five-hour ${ }_{121}$ persister assay might reveal new insights into bacterial ${ }_{122}$ killing dynamics that are relevant for the week-long an- ${ }_{123}$ tibiotics treatment of bacterial infections recommended ${ }_{124}$ by the WHO [1].

Lastly, the molecular mechanism(s) of persister for- ${ }_{126}$ mation is still unknown [18, 12]. Many intracellu- ${ }_{127}$ lar components have been proposed to play a role ${ }_{128}$ $[19,15,20,21,22,23]$, but so far no single mechanism ${ }_{129}$ convincingly explains persister formation. In fact, bacterial persistence presents as a very complex and diverse problem, where the survival fraction could be composed of different subpopulations.

Despite the complexity of persistence, it has been established that stationary phase cultures contain a greater persister fraction than exponentially growing cultures [5]. Stationary phase bacteria may refer to bacteria in a multitude of different physiological states, but is typically associated with starvation stress [24]. Furthermore, the second messenger ( $\mathrm{p}) \mathrm{ppGpp}$, which accumulates during starvation responses was frequently shown to correlate positively with persistence formation $[17,25,5,21]$. Hence, it is critical for persistence research to understand the degree to which (p)ppGpp levels affect persistence, and under which circumstances.

The present study investigates persistence in the balanced exponential growth phase where (p)ppGpp levels are relatively low and correlate inversely with the growth rate. It deals with whether $E$. coli forms spontaneous persisters in the exponential phase, their dependence on the growth rate, how long they survive and how their formation relates to (p)ppGpp levels. We followed the long-term survival of E. coli K12 populations exposed to a lethal concentration of ciprofloxacin for one week. The growth rate of the E. coli population at the time of antibiotics exposure was varied using growth medium with either of two different carbon sources. In addition, a knockout strain was constructed in the wildtype background, removing the gene relA and, thus, introducing a (p)ppGpp synthesis deficiency. Furthermore, we compared the killing dynamics with and without a short carbon-starvation period immediately prior to the killing assay. The starvation pulse had a considerable influence on persister formation and had a very long memory effect on the survival of the population.

\section{Results}

\section{Long-term persister assay of exponentially growing} cells

First, we investigated whether long-term persister cells form during exponential growth in glucose minimal medium. We treated balanced cultures of $E$. coli K-12 with ciprofloxacin and monitored the killing dynamics for one week of antibiotics treatment. Balanced growth was obtained by culturing the cells for more than twenty doubling times in the target medium at 37 ${ }^{\circ} \mathrm{C}$, keeping the cell density of the culture below an $\mathrm{OD}_{436}$ of 0.3 by repeated back-dilutions. Cultures were then treated for a week with $10 \mu \mathrm{g} / \mathrm{mL}$ ciprofloxacin 


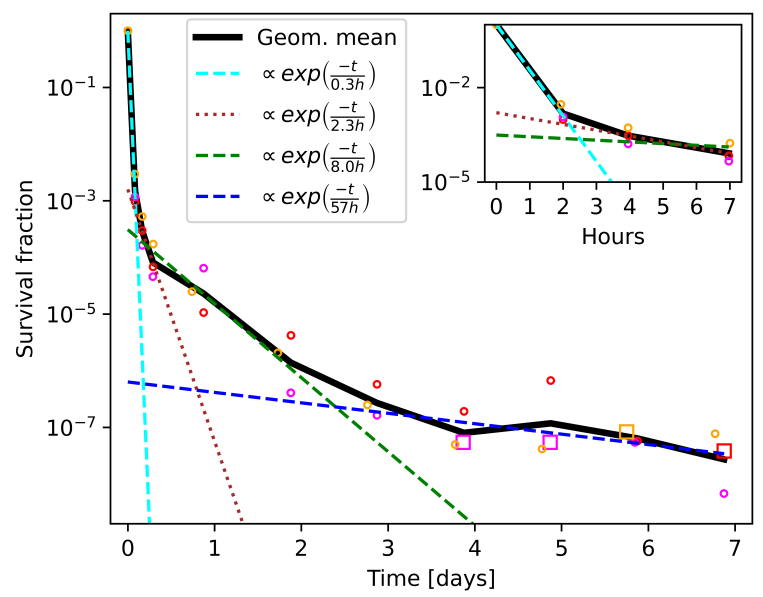

Figure 1: Killing dynamics of exponential phase E. coli persisters. Killing dynamics for exponential phase persisters. The bacteria were grown in glucose minimal medium. All three biological replicates are shown for each data point. The black line is the geometrical mean. Each separate phase of a triphasic fit are shown respectively as cyan, green and blue dashed lines. The second phase of a biphasic fit to the first seven hours is shown as a brown dotted line.

and their killing dynamics were monitored by repeated platings of culture aliquots on antibiotics-free growth medium (See methods for detail).

E. coli persisters were formed during exponential balanced growth in glucose minimal medium, as seen in Fig. 1. There was a fast initial killing at a rate of about $1 / 0.3\left(\mathrm{~h}^{-1}\right)$, with a slower killing rate already after two hours. When we fit a biphasic curve (summation of two exponential functions) to the data up to 7 hours, the second phase of killing is at a rate of about $1 / 2.3\left(\mathrm{~h}^{-1}\right)$, shown by a brown line in the inset in Fig. 1. However, 159 for longer times, this fit significantly underestimates the 160 survival time of the bacterial population (Fig. 1). In ${ }_{161}$ other words, there is an even slower phase of killing, 162 extending from seven hours to four days. Lastly, from ${ }_{163}$ day five to day seven, the remaining cells were killed at ${ }_{164}$ a very slow rate, however, this part of the data is less ${ }_{165}$ reliable due to the small numbers of recovered colonies. ${ }_{166}$

To identify the various phases in a quantitative man- 167 ner, the data was fitted with a sum of exponentials, and ${ }_{168}$ the appropriate number of exponentials was chosen with 169 a $\chi^{2}$ test (see SI Appendix section 2). The test rejects a 170 double exponential as a good fit, but accepts the hypoth- 171 esis of a triple exponential. The best fit obtained was the 172 first phase of killing at a rate $1 / 0.3\left(\mathrm{~h}^{-1}\right)$ (a cyan dashed 173 line), the second phase of killing at a rate of $1 / 8\left(\mathrm{~h}^{-1}\right){ }_{174}$ (a green dashed line), and the third phase of killing at 175 a rate of $1 / 57\left(\mathrm{~h}^{-1}\right)$ (a blue dashed line). The long-term 176 killing dynamics thus had more than two time-scales, 177

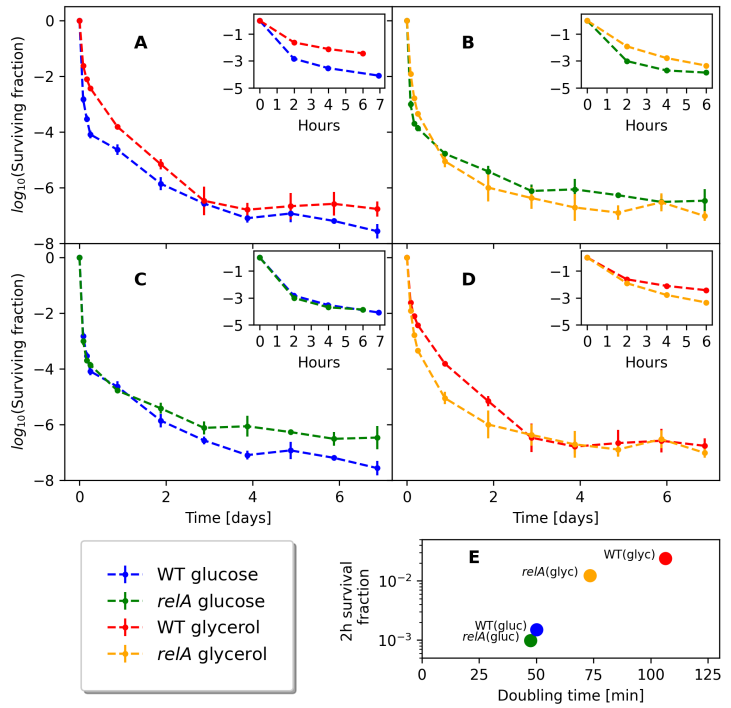

Figure 2: Killing dynamics in different medium with and without the relA gene. Each line represents three biological replicates. (A) The wildtype killing dynamics in either glycerol or glucose minimal medium. (B) The $\triangle$ relA killing dynamics in either glycerol or glucose minimal medium. (C) Killing dynamics in glucose minimal medium for comparison between the wildtype and the $\Delta$ relA strain. The $\triangle$ relA mutant had more survivors than the wildtype from day 4 and later, but there was no statistically significant difference at any of the timepoints (SI Appendix section 2). (D) Killing dynamics in glycerol minimal medium for comparison between the wildtype and the $\Delta$ relA strain. (E) Survival fraction after two hours of ciprofloxacin treatment compared to the growth rate prior to antibiotics treatment.

and the dominant phase of killing is only apparent after several days of measurement.

The exponential phase growth rate determines many aspects of bacterial physiology, including the macromolecular composition $[8,26]$. The growth rate at the time of antibiotics exposure has previously been linked to short-term survival of antibiotics $[10,11]$, and could also affect long-term survival. For that reason, the longterm killing assay was repeated with glycerol as the carbon source, which strongly affected the wildtype growth rate. In glucose minimal medium, the wildtype doubling time was $50 \pm 1.4 \mathrm{~min}$, while it was $106 \pm 3.0 \mathrm{~min}$ in glycerol minimal medium. This difference had an impact on the initial phase of killing for up to seven hours, showing slower killing and a higher survivor fraction in glycerol (Fig. 2A inset). However, the long-term killing curve in glycerol minimal medium was not merely a decelerated version of the killing curve in glucose minimal medium. Though more cells survive in glycerol than in 
glucose for up to two days (Fig. 2A), the survival curve ${ }_{228}$ in glycerol medium at 1 to 2 days had a steeper drop 229 than in glucose medium, resulting in comparable sur- 230 viving fractions after 3 days. In fact, in two of the three ${ }_{231}$ biological replicates of wildtype cultures grown in glyc- 232 erol, almost no survivors were observed after 3 days of ${ }_{233}$ killing (see SI Appendix section 1). Overall, the wild- 234 type killing dynamics in glycerol had more than two time scales, with a best fit of four separate phases of killing (SI Appendix section 2).

\section{Deletion of relA affects the killing dynamics}

In glycerol minimal medium, the steady state 239 (p)ppGpp level of the wildtype strain is higher than in 240 glucose minimal medium [27]. Because the (p)ppGpp 241 level has been frequently associated with persister for- 242 mation, we next aimed at investigating the effect of ${ }_{243}$ the initial (p)ppGpp level on long-term survival. E. ${ }_{244}$ coli encodes the primary (p)ppGpp synthetase RelA 245 and the secondary (p)ppGpp synthetase SpoT, the lat- ${ }_{246}$ ter of which is bifunctional as a (p)ppGpp hydrolase. ${ }_{247}$ The nutrient-dependent steady-state growth rates are ${ }_{248}$ inversely related to the concentrations of (p)ppGpp, 249 both for $\mathrm{relA}^{+}$an $\mathrm{relA}^{-}$strains [27], and for many 250 carbon sources, the growth rates and (p)ppGpp levels 251 of $\mathrm{relA}^{+} / \mathrm{relA}^{-}$strain pairs are indistinguishable due to ${ }_{252}$ (p)ppGpp synthesis by SpoT. However, in low energy ${ }_{253}$ carbon sources like glycerol or acetate, SpoT produces 254 insufficient (p)ppGpp to suppress the growth rate when ${ }_{255}$ RelA is missing, leading to an enhanced growth rate ${ }_{256}$ of the RelA mutant strain relative to the wildtype [27]. 257 We constructed a $\triangle$ relA mutant to clarify the role of ${ }_{258}$ (p)ppGpp in the killing dynamics.

As expected, the difference between the growth rate 260 in glucose and in glycerol minimal medium was smaller 261 for the $\Delta$ relA strain, with a doubling time of $47 \pm 1.5 \mathrm{~min} 262$ in glucose minimal medium and $74 \pm 1.6 \mathrm{~min}$ in glyc- 263 erol minimal medium. The short term (up to 4 hours) 264 survival under antibiotics exposure was also correlated 265 with the growth rate in the $\triangle r e l A$ strain (Fig. 1B inset). 266

Like wildtype cells, the $\triangle$ relA mutant formed long- 267 term survivors in both glucose and glycerol minimal 268 medium with more than two phases of killing (Fig. 2B, 269 see SI Appendix section 2 for statistical analysis). In 270 glucose, the two strains grew at similar rates, and the 271 early killing dynamics of the $\triangle r e l A$ mutant were very 272 similar to that of the wildtype (Fig. 2C). In glycerol, 273 the faster-growing $\triangle$ relA mutant showed a significantly 274 lower level of persisters than the wildtype in the initial 275 phase of killing up to one day (Fig. 2D, see SI Appendix 276 section 2), indicating the importance of growth rate, or 277
ppGpp level, for persister formation in this phase. Interestingly, the long-term survival of the $\triangle$ relA mutant and wildtype were comparable at later times (after 3 days) in glycerol medium, and somewhat higher than the wildtype strain in glucose medium. Thus, survival in the long term is not simply dependent on the population growth rate at the time of antibiotics exposure.

\section{A starvation pulse prior to the antibiotic application af- fects the long-term persistence of wildtype cells in glu- cose minimal medium}

A sudden downshift of the carbon source is known to give a spike of the (p)ppGpp level in the wildtype strain just after the downshift, while the spike is significanlly lower in a RelA strain [28]. We then wondered if a short pulse of carbon source starvation to the exponentially growing cells prior to the killing assay will give a quantifiable difference in the long-term persistence between the wildtype strain and the $\Delta r e l A$ strain.

In order to test this, part of the cultures in balanced growth were filtered into growth medium without a carbon source and starved for 1 hour, before the carbon source was replenished (Figure 3A). Figure 3B shows that the 1-hour starvation pulse resulted in a quick rise of the ppGpp level peaking at about 15 min after the downshift for the wildtype strain in glucose medium, while only a mild increase of the ppGpp level was seen in the $\triangle$ relA strain. Antibiotics were added simultaneously with the carbon source replenishment (Fig. 3A, see methods for details). Remarkably, the short carbon starvation prior to the addition of antibiotics had long-term effects on the killing dynamics. This was especially visible for the wildtype strain grown in glucose minimal medium, where the brief starvation period reproducibly resulted in almost 100 -fold more persisters for up to four days. The difference was abolished by removing relA, as seen in Fig. $4 \mathrm{~B}$; the $\triangle$ relA strain only exhibited increased survival for the first six hours following starvation. As such, the long-term memory of the starvation pulse is seemingly a relA-dependent effect. However, the $\triangle$ relA strain in the steady state growth in glucose had more long-term survivors than the wild-type strain, and the downshift brought the wildtype strain survival fraction to a level similar to the $\Delta$ relA strain.

The effect of the downshift was smaller in the glycerol medium (Fig. 4CD). In the wildtype strain, the average persister level with downshift was higher up to 4 days, but the statistical significance of the difference was confirmed only up to 7 hours due to the larger data scatter for later time points (Fig. 4C). The effect of 

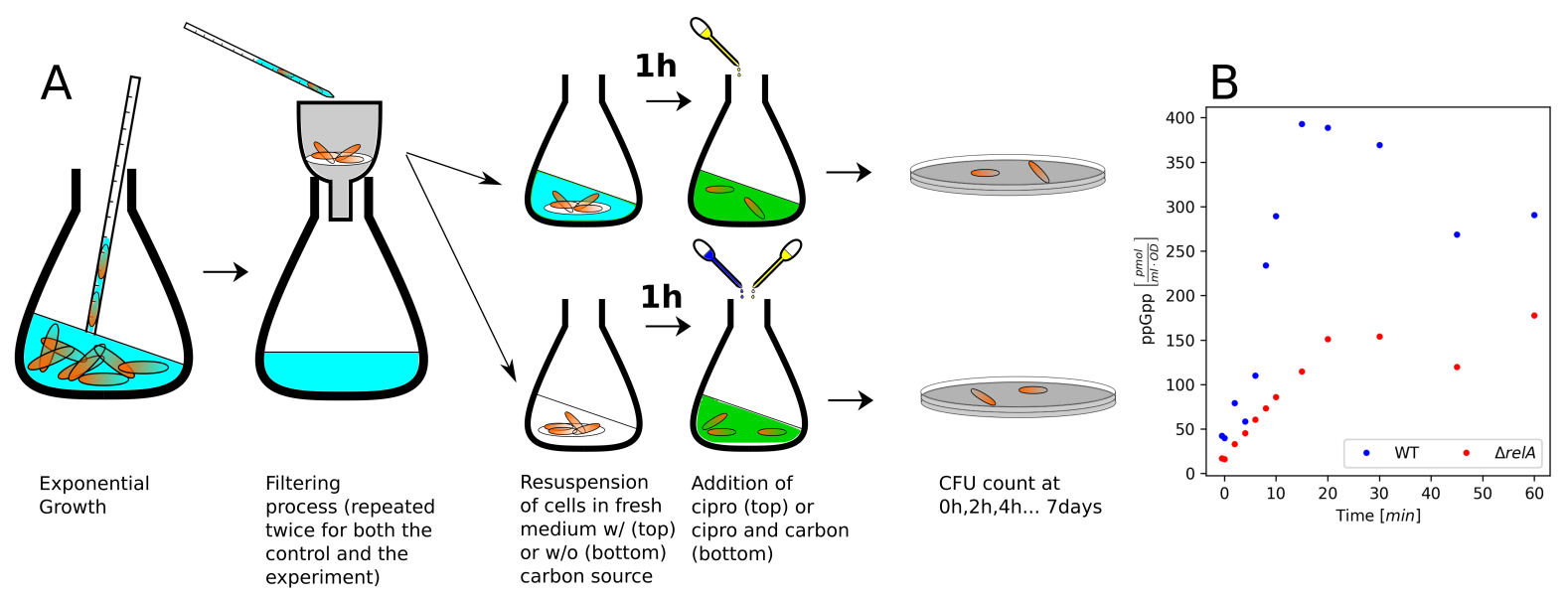

Figure 3: The experimental setup for the long-term persistence assay. (A) Cells were in balanced exponential growth prior to the killing assay. A part of the culture was filtered and then resuspended in medium with or without the carbon source, and incubated for 1 hour. After 1 hour, the ciprofloxacin was added to both of the samples, and at the same time the carbon source was added to the the culture that has been starved fro 1 hour. The first sample was taken just before the additions. After that, samples were taken at 2,4,6/7, 21+24.n hours after the antibiotic addition for $n \in[0 ; 6]$. The samples were washed and plated on agar plates containing the target medium. (B) The 1-hour time course of ppGpp level for the culture grown in the glucose medium, filtered, and resuspended in fresh medium without carbon source. The time zero is the time of the resuspension. The wild type (blue circles) shows clear peak around 15 minutes after the resuspension. $\Delta$ relA strain shows only mild increase of the ppGpp level.

downshift disappeared faster in the $\triangle r e l A$ strain already after 2 days (Fig. 4D).

\section{Discussion}

We expanded the understanding of bacterial killing dynamics with a new long-term persister assay. The use of minimal medium facilitated the formation of longterm persisters during exponential growth, in contrast to growth and killing in rich medium [7]. Spontaneous persisters were observed during the exponential growth phase, both in glucose and glycerol minimal medium, and in some cases they survived at least one week. This long-term survival does not require relA, although the residual (p)ppGpp synthesized by SpoT is likely necessary. In fact, there is an increase in long-term survival of the $\Delta$ relA mutant in glucose.

We have shown that a one-hour starvation pulse prior to the addition of the antibiotic affects long-term survival. This finding is consistent with a previous study of a temporal nitrogen downshift prior to antibiotics treatment, which was shown to elevate the persister level at 24 hours in a relA dependent manner [29]. Our study demonstrated that the memory can be remarkably longlasting, as one-hour carbon starvation gave an increase in survival for at least 4 days in the wildtype strain grown in glucose medium. The molecular mechanism underlying this long-term memory is yet to be inves-

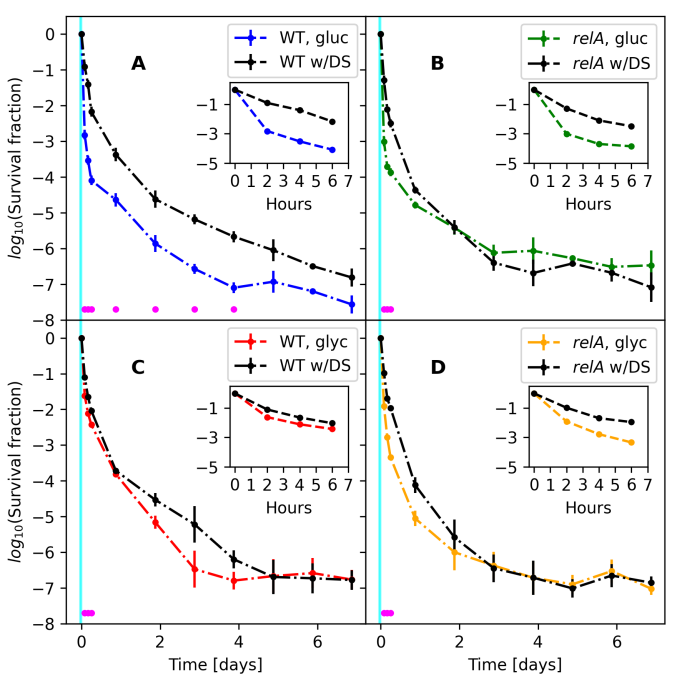

Figure 4: The effect of a short starvation-pulse on the survival dynamics. All strains and conditions are shown with and without the downshift. The black line is with the downshift, the colored line is without the downshift. The magenta dots represent statistically different datapoints as tested by an unequal variances two-sided t-test. The cyan interval illustrates the downshift period prior to the killing assay. (A) The wildtype in glucose. A downshift in glucose significantly enhances wildtype survival for four days and seems to be increased for up to all seven days. (B) The $\triangle$ relA mutant in glucose. (C) The wildtype in glycerol. (D) The $\Delta$ relA mutant in glycerol. 
tigated, but in all likelihood it is linked to the abrupt 355 RelA-mediated rise in (p)ppGpp upon starvation, since the starvation-pulse effect on long-term survival was ${ }^{356}$ abolished in the $\triangle$ relA mutant. In further support of this ${ }^{357}$ hypothesis, there was no long-term effect when glyc- ${ }^{358}$ erol was used as the carbon source, which could be due 359 to the high basal level of (p)ppGpp in glycerol relative 360 to glucose minimal medium [8]. The sensitivity of the survival fraction to the rather short perturbation may be ${ }^{361}$ consistent with the idea that there is a threshold in some 362 molecule concentration to determine if the cell becomes 363 persister or not [30,31], since a small perturbation can 364 have a major impact on the occurrence of rare expres- 365 sion patterns that exceed a extreme threshold [32]. This 366 observation also alerts us that small perturbations in the ${ }_{367}$ experimental procedure may strongly affect the result of 368 persister assays.

This study shows that the long-term killing of E. coli in ciprofloxacin is not adequately described by biphasic dynamics. At least three phases of killing were present in the data. Thus, despite the emphasis on a biphasic behaviour to define persistence [4], we propose a third, or even fourth, phase of killing that may even be more clinically relevant. The presence of additional phases also means that the population survival time will be underestimated by predictions from the biphasic killing assumption. Indeed, it is not sufficient to measure killing dynamics for only five hours and then extrapolate the population survival time from there.

The population growth rate was found to be positively correlated with the killing rate in the initial phase. However, the correlation was diminished in the longer term survival, and lost in the third phase of killing. In shorter persister assays, a difference in growth rate, such as between different mutants, might strongly confound results when analyzing persistence fractions. These differences seem smaller and less relevant in later phases of killing.

This investigation of long-term killing dynamics has 392 added to the concept of bacterial persistence as a com- ${ }^{393}$ plex phenomenon. During long-term antibiotics treat- 394 ment, different mechanisms could account for bacterial 395 survival on different timescales (an hour, a day, a week), 396 although the (p)ppGpp level at the time of initial expo- 397 sure to the antibiotic seems to be important in all cases. 398 As such, persistence seems to be a time-dependent phe- 399 nomenon, where different survival mechanisms account 400 for different bacterial life spans. The presence of several phases in the killing dynamics begs the question if 401 a more extended concept should replace the simplified 402 concept of bacterial persistence.

\section{Methods and Materials}

\section{Strains}

The strain MAS1081 (MG1655 rph ${ }^{+} \mathrm{gatC}^{+} \mathrm{glpR}^{+}$) was used as the wildtype [33]. The $\Delta$ relA, MAS1191, is MAS1081 made $\Delta$ relA251 :: Kan by P1 transduction from CF1651 [34] followed by selection on kanamycin.

\section{Long-term killing assay}

A single colony of the E. coli strain grown on an agar plate was incubated overnight in the target medium (MOPS minimal medium with either glucose or glycerol as the carbon source [35]. See the SI Appendix section 1 for the recipe). The overnight culture was diluted $1: 10^{7}$ in $10 \mathrm{~mL}$ target medium in a $100 \mathrm{~mL}$ Erlenmeyer flask. The flask was continuously shaken at 160 RPM in a $37^{\circ} \mathrm{C}$ room. Hours later, the culture was diluted further, at least $1: 10^{2}$, in $100 \mathrm{~mL}$ preheated medium in a $1 \mathrm{~L}$ Erlenmeyer flask, reaching a total dilution of at least $1: 10^{9}$. Once the $\mathrm{OD}_{436}$ reached a detectable level of 0.01 , at least 5 samples were measured at different timepoints to establish a growth rate. The $\mathrm{OD}_{436}$ was consistently kept below 0.3 . A part of the culture, typically around $20 \mathrm{~mL}$, was filtered, and the cells on the filter were resuspended in $40 \mathrm{~mL}$ of the target medium (w/o carbon source) in a $300 \mathrm{~mL}$ Erlenmeyer flask. As such, both the control and the starved culture were exposed to filtration and resuspension. The medium volume never exceeded 14 percent of the flask volume. The starvation was verified by measuring $\mathrm{OD}_{436}$, to confirm either increase in biomass (control) or no growth (starving culture). See the SI Appendix section 1 for confirmation of the downshift. After one hour of starvation, a sample was taken immediately before ciprofloxacin (10 $\mu \mathrm{g} / \mathrm{mL}$ ) was added along with the carbon source, that was replenished to end the downshift. Samples were taken at times $2,4,6 / 7,21+24 \cdot n$ hours for $n \in[0 ; 6]$. The samples were put on ice for a few minutes and then centrifuged for ten minutes at $4^{\circ} \mathrm{C}$ at $10,000 \mathrm{~g}$. The supernatant was removed and the cell pellet was resuspended in room temperature MOPS buffer with no supplements. The sample was diluted appropriately, never more than 1 : 100 per step, corresponding to $10 \mu \mathrm{L}$ in $990 \mu \mathrm{L}$. The sample was plated with $200 \mu \mathrm{L}$ per plate on minimal medium plates containing the target medium. The plates were kept at $37^{\circ} \mathrm{C}$ for at least one week and all colonies were counted. The whole experiment is illustrated in figure 3.

\section{Controls}

After each completed experiment (7 days), $200 \mu \mathrm{L}$ of culture, still containing antibiotics, was spread on a 
plate with the target medium. This was left at $37^{\circ} \mathrm{C}$ for ${ }_{452}$ at least 7 days, and no growth confirmed the absence of ${ }_{453}$ a growing resistant culture in the flask. In addition, the 454 activity of the antibiotics in the culture was tested after 455 seven days by dropping $20 \mu \mathrm{L}$ on a lawn of growing $E$. coli.

\section{Analysis of time-scales in the killing dynamics}

Time-scales in the killing dynamics were statistically 458 identified by fitting a sum of exponential functions to 459 the data. The model with the least number of param- 460 eters, that could not significantly be rejected, was then 461 chosen [36]. The functional form of the models was a sum of exponential functions. The number of exponential functions in the sum corresponded to the number of time-scales. A biphasic killing curve would for exam- 463 ple be well fitted by the sum of two exponentials. The ${ }^{464}$ $\chi^{2}$-test for goodness of fit is used for identifying the ap- ${ }^{465}$ propriate model using the Minuit minimization software ${ }^{466}$ $[37,38]$ (see the SI Appendix section 2 for specifics of ${ }_{468}$ the analysis).

\section{Statistical analysis}

All killing curves are based on three biological repli- ${ }^{47}$ cates. The mean is determined as the mean of the log- ${ }_{475}$ arithmic values, which corresponds to the geometric 476 mean. This is done to get a more adequate mean-value ${ }^{477}$ representation in log-space. The uncertainties are also ${ }_{479}^{478}$ calculated as the standard deviations of the log trans- ${ }_{480}^{479}$ formed values. Whenever a datapoint had the value ${ }_{481}$ zero, which happened frequently, that value was re- ${ }^{482}$ placed with the detection limit, to get a sensible value ${ }^{48}$ in log-space. An unequal variance two-sided t-test was ${ }_{485}{ }^{484}$ used to determine significant differences between two ${ }_{486}$ datapoints at the same timepoint $(P<0.05)$.

\section{ppGpp measurements}

Cultures were grown for two generations in the pres- 49 ence of $75 \mu \mathrm{Ci} / \mathrm{mL} 32 \mathrm{P}$-phosphate at a total phosphate ${ }_{493}^{492}$ concentration of $0.33 \mathrm{mM}$. At the time of starvation, 494 cultures were filtered, washed in medium without glu- 495 cose and phosphate and resuspended in medium with- ${ }^{496}$ out glucose but containing 32P at the same specific ac- ${ }_{498}$ tivity as during growth. These steps were performed at 499 $37^{\circ} \mathrm{C}$ and lasted less than 2 min. For determination of 500 the nucleotide pools, $100 \mu \mathrm{L}$ of culture was harvested ${ }_{502}^{501}$ into $20 \mu \mathrm{L} 2 \mathrm{M}$ formic acid at $0^{\circ} \mathrm{C}$. After centrifuga- ${ }_{503}$ tion the nucleotides in the supernatant were separated by 504 chromatography on polyethyleneimine-cellulose plates. ${ }^{505}$ The activities of the individual spots were quantified ${ }_{507}^{506}$ by PhosphoImager scans (Typhoon Phosphor Imager 508
FLA7000 (GE Healthcare)) of the plates. The specific activity of the signal was determined from a medium sample from the individual cultures spotted onto the same brand of plates.

\section{Acknowledgments}

MSS and NM thank S. Semsey for fruitful discussions. This work was supported by the Danish National Research Foundation (DNRF120) and the Independent Research Fund Denmark (8049-00071B and 8021-00280A).

\section{References}

[1] M. D. Pezzani, G. Be, P. Cattaneo, A. Zaffagnini, F. Gobbi, P. Rodari, Z. Bisoffi, E. Tacconelli, Evidence based review on optimal duration of antibiotic therapy for bacterial infections to support antimicrobial stewardship recommendations WHO Secretariat Nicola Magrini, Secretary of the Expert Committee on Selection and Use of Essential Medicines; In (2019) 1-28.

[2] J. W. Bigger, Treatment of Staphylococcal Infections With Penicillin By Intermittent Sterilisation, The Lancet 244 (1944) 497500. doi:10.1016/S0140-6736(00)74210-3.

[3] N. Q. Balaban, J. Merrin, R. Chait, L. Kowalik, S. Leibler, Bacterial Persistence as a Phenotypic Switch, Science 305 (2004) 1622-1625. doi:10.1126/science.1099390.

[4] N. Q. Balaban, S. Helaine, A. Camilli, J. J. Collins, J.-M. Ghigo, W.-D. Hardt, A. Harms, M. Heinemann, Definitions and guidelines for research on antibiotic persistence, Nature Reviews Microbiology 17 (2019) 441-448. URL: http://dx.doi.org/10.1038/s41579-019-0196-3. doi:10.1038/s41579-019-0196-3.

[5] A. Harms, C. Fino, M. A. Sørensen, S. Semsey, K. Gerdes, Prophages and Growth Dynamics Confound Experimental Results with Antibiotic-Tolerant Persister Cells, mBio 8 (2017) 1-18. doi:10.1128/mBio.01964-17.

[6] A. Brauner, O. Fridman, O. Gefen, N. Q. Balaban, Distinguishing between resistance, tolerance and persistence to antibiotic treatment, Nature Reviews Microbiology 14 (2016) 320-330. URL: http://dx.doi.org/10.1038/nrmicro.2016.34. doi:10.1038/nrmicro.2016.34.

[7] I. Keren, N. Kaldalu, A. Spoering, Y. Wang, K. Lewis, Persister cells and tolerance to antimicrobials, FEMS Microbiology Letters 230 (2004) 13-18. doi:10.1016/S0378-1097(03)00856-5.

[8] H. Bremer, P. P. Dennis, Modulation of Chemical Composition and Other Parameters of the Cell by Growth Rate, Escherichia coli and Salmonella: cellular and molecular biology 2 (1987) 1527-1542. doi:10.1046/j.1365-2486.2000.06017.x.

[9] M. Scott, C. W. Gunderson, E. M. Mateescu, Z. Zhang, T. Hwa, American Association for the Advancement of Science, Science 330 (2010) 1099-1102. doi:https://doi.org/10.1126/science.1192588.

[10] E. Tuomanen, R. Cozens, W. Tosch, O. Zak, A. Tomasz, The rate of killing of Escherichia coli by beta-lactam antibiotics is strictly proportional to the rate of bacterial growth., Journal of general microbiology 132 (1986) 1297-304. doi:10.1099/00221287-132-5-1297.

[11] A. J. Lee, S. Wang, H. R. Meredith, B. Zhuang, Z. Dai, L. You, Robust, linear correlations between growth rates and $\beta$-lactam mediated lysis rates (2018) 1-6. doi:10.1073/pnas.1719504115. 
[12] M. H. Pontes, E. A. Groisman, Slow growth determines non- 574 heritable antibiotic resistance in Salmonella enterica, Science 575 Signaling 12 (2019) 1-11. doi:10.1126/scisignal.aax3938. 576

[13] E. M. Windels, Z. B. Meriem, T. Zahir, K. J. Ver- 577 strepen, P. Hersen, B. V. den Bergh, J. Michiels, Iso- 578 lation of persisters enabled by B-lactam-induced fila- 579 mentation reveals their single-cell awakening character- 580 istics, Communications Biology (2019) 600700. URL: 581 https://www.biorxiv.org/content/10.1101/600700v1. 582 doi:10.1101/600700.

[14] E. Simsek, M. Kim, Power-law tail in lag time dis- 584 tribution underlies bacterial persistence 116 (2019). 585 doi:10.1073/pnas.1903836116.

[15] F. Goormaghtigh, N. Fraikin, M. Putrins, T. Hallaert, V. Hau- 587 ryliuk, A. Garcia-Pino, A. Sjödin, S. Kasvandik, K. Udekwu, 588 T. Tenson, N. Kaldalu, L. Van Melderen, Reassessing the Role 589 of Type II Toxin-Antitoxin Systems in Formation of Escherichia 590 coli Type II Persister Cells, mBio 9 (2018) 1-14.

[16] F. Goormaghtigh, L. V. Melderen, Single-cell imaging and char- 592 acterization of Escherichia coli persister cells to ofloxacin in ex- 593 ponential cultures, Science Advances 5 (2019).

[17] S. Helaine, A. M. Cheverton, K. G. Watson, L. M. Faure, S. A. 595 Matthews, D. W. Holden, Internalization of Salmonella by 596 Macrophages Induces Formation of Nonreplicating Persisters, 597 Science 343 (2014) 204-209.

[18] A. Harms, E. Maisonneuve, K. Gerdes, Mechanisms of bacterial 599 persistence during stress and antibiotic exposure, Science 354600 (2016). doi:10.1126/science.aaf4268.

[19] E. A. Zalis, A. S. Nuxoll, S. Manuse, G. Clair, L. C. Radlin- 602 ski, B. P. Conlon, J. Adkins, K. Lewis, Stochastic Variation in 603 Expression of the Tricarboxylic Acid Cycle Produces Persister 604 Cells, mBio 10 (2019) 1-10.

[20] Y. Shan, A. B. Gandt, S. E. Rowe, J. P. Deisinger, B. P. Conlon, 606 K. Lewis, ATP-Dependent Persister Formation in Escherichia 607 coli, mBio 8 (2017).

[21] M. S. Svenningsen, A. Veress, A. Harms, N. Mitarai, S. Sem- 609 sey, Birth and Resuscitation of ( p ) ppGpp Induced An- 610 tibiotic Tolerant Persister Cells, Scientific Reports 9 (2019). 611 doi:10.1038/s41598-019-42403-7.

[22] R. Yamasaki, S. Song, M. Benedik, T. Wood, Persis- 613 ter Cells Resuscitate Using Membrane Sensors that Acti- 614 vate Chemotaxis, Lower cAMP Levels, and Revive Ri- 615 bosomes, SSRN Electronic Journal 23 (2019) 100792. 616 URL: https://doi.org/10.1016/j.isci.2019.100792. 617 doi: $10.2139 /$ ssrn.3399578

[23] R. C. Molina-Quiroz, C. Silva-Valanzuela, J. Brewster, E. Castro-Nallar, S. B. Levy, A. Camilli, Cyclic AMP Regulates Bacterial Persistence through Repression of the Oxidative Stress Response and SOS-Dependent DNA Repair in Uropathogenic Escherichia coli, mBio 9 (2018) e02144-17. doi:10.1128/mBio.02144-17.

[24] T. Nyström, Stationary-Phase Physiology, Annual Review of Microbiology 58 (2004) 161-181. doi:10.1146/annurev.micro.58.030603.123818

[25] J. L. Radzikowski, H. Schramke, M. Heinemann, Bacterial persistence from a system-level perspective, Current Opinion in Biotechnology 46 (2017) 98-105. URL: http://dx.doi.org/10.1016/j.copbio.2017.02.012. doi:10.1016/j.copbio.2017.02.012.

[26] S. Hui, J. M. Silverman, S. S. Chen, D. W. Erickson, M. Basan, J. Wang, T. Hwa, J. R. Williamson, Quantitative proteomic analysis reveals a simple strategy of global resource allocation in bacteria, Molecular Systems Biology 11 (2015) e784-e784. doi: $10.15252 / \mathrm{msb} .20145697$.

[27] J. Ryals, R. Little, H. Bremer, Control of rRNA and
tRNA syntheses in Escherichia coli by guanosine tetraphosphate, Journal of Bacteriology 151 (1982) 1261-1268. doi:10.1128/jb.151.3.1261-1268.1982.

[28] R. A. Lazzarini, M. Cashel, J. Gallant, On the regulation of guanosine tetraphosphate levels in stringent and relaxed strains of Escherichia coli., Journal of Biological Chemistry 246 (1971) 4381-4385.

[29] D. R. Brown, Nitrogen starvation induces persister cell formation in Escherichia coli, Journal of Bacteriology 201 (2019). doi:10.1128/JB.00622-18.

[30] E. Rotem, A. Loinger, I. Ronin, I. Levin-Reisman, C. Gabay, N. Shoresh, O. Biham, N. Q. Balaban, Regulation of phenotypic variability by a threshold-based mechanism underlies bacterial persistence, Proceedings of the National Academy of Sciences of the United States of America 107 (2010) 12541-12546. doi:10.1073/pnas.1004333107.

[31] J. E. Michiels, B. Van den Bergh, N. Verstraeten, J. Michiels, Molecular mechanisms and clinical implications of bacterial persistence, Drug Resistance Updates 29 (2016) 76-89. doi:10.1016/j.drup.2016.10.002.

[32] M. S. Svenningsen, S. Semsey, N. Mitarai, Gene Expression Changes with Minor Effects on the Population Average Have Major Effects on the Occurrence of Cells with Extreme Protein Concentrations, mSphere 4 (2019) 1-6. doi:10.1128/msphere.00575-18.

[33] B. Gummesson, S. A. Shah, A. S. Borum, M. Fessler, N. Mitarai, M. A. Sørensen, S. L. Svenningsen, Valine-induced isoleucine starvation in escherichia coli k-12 studied by spike-in normalized rna sequencing, Frontiers in genetics 11 (2020) 144.

[34] H. Xiao, M. Kalman, K. Ikehara, S. Zemel, G. Glaser, M. Cashel, Residual guanosine 3',5'-bispyrophosphate synthetic activity of relA null mutants can be eliminated by spoT null mutations, Journal of Biological Chemistry 266 (1991) 5980-5990.

[35] F. C. Neidhardt, P. L. Bloch, D. F. Smith, Culture medium for enterobacteria, Journal of Bacteriology 119 (1974) 736-747. doi:10.1128/jb.119.3.736-747.1974.

[36] R. J. Barlow, STATISTICS: A GUIDE AND REFERENCE TO THE USE OF STATISTICAL METHODS IN THE PHYSICAL SCIENCES, Wiley, 1993.

[37] F. James, M. Roos, Minuit - A System for Function Minimization and Analysis of the Parameter Errors and Correlations, Computer Physics Communications 10 (1975) 343-367.

[38] iminuit - A Python interface to Minuit, 2020. 